\title{
Caracterización del perfil pedagógico de los docentes e instructores de la Escuela de Aviación del Ejército Nacional y la Escuela Militar de Suboficiales "Sargento Inocencio Chincá"1
}

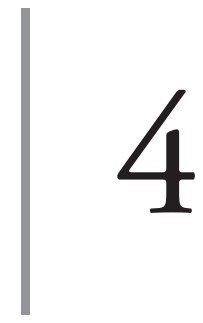

https://doi.org/10.21830.9789585350663.04

\author{
Martha Patricia Vives Hurtado \\ Diana Carolina Contreras Gutiérrez \\ Escuela de Aviación del Ejército \\ Yerife Andrea Parra Orozco ${ }^{4}$
}

Escuela Militar de Suboficiales "Sargento Inocencio Chincá”

\begin{abstract}
Resumen. La formación militar del Ejército Nacional se realiza en las escuelas de formación para aspirantes a oficiales y suboficiales, y en las escuelas de capacitación para fortalecer las competencias de los integrantes de la institución. En la formación y capacitación intervienen personal civil y militar. El objetivo de esta investigación es caracterizar el perfil pedagógico de los docentes e instructores de la Escuela Militar de Suboficiales "Sargento Inocencia Chincá" (Emsub) y de la Escuela de Aviación del Ejército Nacional (EsAVE), con el fin de conocer su formación pedagógica, experiencia docente y producción investigativa, y, a partir de dicha información, proponer planes de formación y actualiza-
\end{abstract}

1 Este capítulo presenta los resultados del proyecto de investigación "Caracterización del perfil pedagógico de los profesores e instructores de las Escuelas de Formación y Capacitación del Ejército Nacional", del grupo de investigación en Aviación Militar de la Escuela de Aviación del Ejército (Esave), categorizado en C por Minciencias y con código de registro COL0077618, en colaboración con el grupo de investigación InTEMIL de la Escuela Militar de Suboficiales "Sargento Inocencio Chincá", registrado con el código COL0151849 de Minciencias. Los puntos de vista pertenecen a los autores y no reflejan necesariamente los de las instituciones participantes.

2 Psicóloga de la Universidad Católica de Colombia. Magíster en Educación de la Pontificia Universidad Javeriana. Doctora en Sociología Jurídica e Instituciones Políticas de la Universidad Externado de Colombia. Investigadora de la Escuela de Aviación del Ejército. OrCID: https://orcid.org/0000-00017795-1494 - Contacto: marthaviveshurtado@cedoc.edu.co

3 Administradora ambiental de la Universidad Distrital Francisco José de Caldas. Especialista en Formulación y Evaluación de Proyectos de la Universidad Católica de Colombia. Magíster en Desarrollo y Gerencia de Proyectos de la Escuela Colombiana de Ingeniería. Investigadora de la Escuela de Aviación del Ejército Nacional. ORCID: https://orcid.org/0000-0002-8996-5348 - Contacto: dianacontrerasgutierrez@ cedoc.edu.co

4 Licenciada en Filosofía y Letras de la Universidad de Caldas. Magíster en Educación de la Universidad del Tolima. Docente e investigadora de la Unidad de Investigación de la Escuela Militar de Suboficiales "Sargento Inocencio Chinca”". Orcid: https://orcid.org/0000-0002-1670-3769 - Contacto: Yerife.parra.docente@emsub.edu.co 
ción docente en las escuelas. Para realizar la investigación se usó el método cuantitativo y se diseñó una encuesta para recolectar la información, con una muestra de 89 docentes-instructores. Los resultados evidencian que los docentes en su mayoría son civiles, con contratación por hora cátedra y con título profesional. Reconocen que es necesario seguir formándose para brindar una educación de calidad e identifican temas que van desde las estrategias didácticas, el uso de las Tic, los tipos y las estrategias de evaluación, hasta la actualización de temas disciplinarios y militares.

Palabras clave: docente; formación; instructor; pedagogía; perfil.

\section{Introducción}

Actualmente, se habla de la importancia de la calidad de la educación y se reconoce que los docentes e instructores son un factor fundamental. El perfil de los docentes o instructores debe ser pertinente a los programas académicos y asignaturas que ofrece una institución educativa. Sin embargo, cuando se contrata a un profesor se da mayor importancia o énfasis a su conocimiento disciplinar o experticia, es decir, si es ingeniero ambiental, se espera que domine los temas de esa ingeniería, pero poco se pregunta por su saber pedagógico y didáctico. En otras palabras, cuando se habla de la formación pedagógica y didáctica del docente, se hace referencia a si tiene el conocimiento y las herramientas para enseñar el conocimiento disciplinar que posee.

La educación y la formación del Ejército Nacional es direccionada por el Ministerio de Defensa Nacional a través del Plan Estratégico del Sistema Educativo de las Fuerzas Armadas (PesE). Específicamente, el Ejército Nacional cuenta con escuelas de formación y escuelas de capacitación: las escuelas de formación realizan la incorporación para formar oficiales y suboficiales, mientras que las escuelas de capacitación realizan la preparación de oficiales y suboficiales para fortalecer sus competencias según la especialidad del militar.

El Ministerio de Defensa reestructura en el Pese la educación de las Fuerzas Armadas considerando como uno de los pilares fundamentales la formación profesional militar y policial inspirada en la doctrina (Ministerio de Defensa, 2008). El Ministerio de Defensa estableció el Pese para el periodo 2007-2019 y define el Sistema Educativo de las Fuerzas Armadas (SEFA) como el conjunto de elementos interrelacionados en un círculo virtuoso de formar, actualizar, capacitar, instruir y entrenar a los integrantes de las Fuerzas Armadas. La competencia se define en el marco educativo de las Fuerzas Militares como 
el elemento que integra aspectos que tienen que ver con principios, valores y virtudes militares, conocimiento; habilidad y destrezas; actitudes o comportamientos; es decir, abarca aspectos de tipo ético, cognitivo, procedimental y actitudinal. Interrelacionados en la búsqueda de desempeńo eficiente en entornos relacionados con la profesión militar. (Ministerio de Defensa Nacional, 2010, p. 26)

En el 2010, el Ministerio de Defensa redimensionó los currículos en la política educativa de las Fuerzas Militares e implementó el desarrollo de competencias orientadas a la formación de los estudiantes en cuanto al ser, el saber, el hacer y el convivir:

En el ámbito del Proyecto Educativo de las Fuerzas Armadas (Pefa) no es preocupación única la formación militar o policial, sino la formación integral: ciudadanos con una sólida formación en principios, valores, virtudes y ética profesional; ejercitados en el arte de pensar, con el desarrollo del pensamiento complejo para alcanzar un sólido criterio militar o policial; profesionales con excelencia en la formación humanística, y ciudadanos ejemplares orientados al servicio de la comunidad; es decir, se trata de llevar a cabo una profunda transformación en la formación de las "competencias del ser". (Ministerio de Defensa, 2008, p. 17)

En el marco educativo de las Fuerzas Militares la competencia se define como

el elemento que integra aspectos que tienen que ver con principios, valores y virtudes militares, conocimiento; habilidad y destrezas; actitudes o comportamientos; es decir, abarca aspectos de tipo ético, cognitivo, procedimental y actitudinal. Interrelacionados en la búsqueda de desempeño eficiente en entornos relacionados con la profesión militar. (Ministerio de Defensa, 2010, p. 26)

Los decretos 1070 del 2015 y 1495 de 2002 definen las cincos categorías de profesor militar y señalan que los profesores serán de tiempo completo o de tiempo incompleto, cualquiera que sea su categoría. Igualmente, en el perfil militar se establecen las dimensiones de la formación integral del militar y sus competencias. Específicamente, se establecen tres dimensiones: (1) Como ser pensante y cognoscente, capacidades intelectuales, metodológicas, investigativas y metacognitivas. (2) Como ser social y axiológico, capacidades de interacción de comunicaciones, de convivencia, el desarrollo de actitudes y valores. 
(3) Como ser práctico, desarrollo de habilidades y destrezas, el dominio de unas prácticas y la intervención en medios y contextos específicos. Asimismo, se establecen cuatro competencias generales: ser, hacer, saber y convivir. Cada una de ellas contiene un conjunto de competencias específicas que se deben desarrollar y fomentar en los programas ofertados (Ministerio de Defensa Nacional, 2010).

Cabe destacar que el Ejército Nacional desarrolla un proyecto para integrar una sola institución de educación direccionada por el Comando de Educación y Doctrina (CEDOc), que tiene como objeto realizar procesos educativos encaminados a la formación, la capacitación, la instrucción, el entrenamiento y el reentrenamiento para el perfeccionamiento del talento humano, de tal manera que se proyecte una doctrina acorde con las exigencias globales (CEDOC, 2020). El CEDoc guía los procesos curriculares de las escuelas de formación y capacitación del Ejército Nacional con los lineamientos del Sefa y el Pefa.

\section{Marco teórico}

Según Moscoso y Hernández (2015), la formación pedagógica de los profesores de instituciones de educación superior (IEs) es una preocupación relativamente reciente, cuyo inicio se remonta a la década del sesenta. En esa década se comienza un proceso de reflexión sobre la formación pedagógica de los docentes de las Ies, ya que la formación profesional se centra en las disciplinas, y los profesionales que se dedican laboralmente a la docencia poseen pocos conocimientos relativos a la didáctica para la enseñanza de sus asignaturas. Los profesores que no tienen una formación pedagógica tienen dificultad para comprender el modelo pedagógico del proyecto educativo institucional (PeI) o del currículo del programa en el que están inmersos, no saben cómo formular los objetivos de aprendizaje, no logran seleccionar los métodos, las herramientas y/o las estrategias de enseñanza más apropiados para desarrollar sus clases, presentan formas de evaluación tradicional, entre otros aspectos. Por tanto, los docentes muchas veces realizan su quehacer a partir de su conocimiento intuitivo y empírico, que han heredado de sus antiguos profesores. Según González y Malagón (2015),

la pedagogía y la didáctica son fundamentales en los procesos de formación de los profesores universitarios. Estas áreas proveen elementos para una adecuada 
construcción del conocimiento con los estudiantes. La formación de los profesores universitarios es un tema fundamental a la hora de pensar en la calidad de la enseńanza de los profesionales y cualificar el aporte del recurso humano en la sociedad. (p. 291)

Estos investigadores realizaron una indagación sobre la formación pedagógica y didáctica de los docentes en Colombia, a partir de la cual plantean que las universidades privadas son las que más se han preocupado por este tipo de formación, con cursos cortos y programas de especialización, maestría y doctorado en temáticas relacionadas con la educación, de manera que en algunas universidades se presentan programas de cualificación docente.

Igualmente, plantean que los docentes de las IEs requieren procesos de formación y capacitación en educación por competencias, calidad educativa, procesos de acreditación, las nuevas formas de acceder a la información, exigencias del mundo globalizado en sus campos de conocimiento, didácticas de las ciencias, ética del profesor universitario, desarrollo de habilidades científicas y tecnológicas en sus estudiantes, entre otros temas (González \& Malagón, 2015).

Por su parte, María de la Cruz Tomé (citada por Moscoso \& Hernández, 2015) opina que la formación pedagógica del profesor universitario es necesaria para profesionalizar la labor docente y reconocer que su tarea es compleja, difícil y retadora. Eso exige tener presente la relación entre la formación docente y la calidad educativa, así como la "complementariedad" entre la formación de las disciplinas por enseñar y los procedimientos para facilitar su aprendizaje.

Por lo anterior, es necesaria la formación pedagógica y didáctica de los docentes e instructores en los procesos educativos, pero ¿qué se entiende por saber pedagógico? De acuerdo con Zambrano (2005; citado por González \& Ospina, 2013),

se concibe como una construcción, un conocimiento frente al hecho educativo que no solo tiene en cuenta el aspecto práctico, sino su fundamentación teórica [...]. En este sentido, el saber pedagógico lo constituye un conjunto de elementos teórico-prácticos que, en un ejercicio reflexivo, configuran un tipo de saber a partir de las prácticas de enseñanza, en las cuales confluye el saber y la interacción social, con miras a la elaboración de un proyecto social. (p. 97)

En la formación militar, el papel que el docente cumple frente a las exigencias y demandas del mundo y sus tendencias tecnológicas implica que siga de cerca su desarrollo y su dominio en el aula involucrando actitudes, 
estrategias y recursos novedosos (Mantilla et al., 2020). Cabe señalar, además, que las áreas de formación de las Fuerzas Militares son parte del sistema educativo del país, de manera que acopla sus procesos académicos a los criterios de calidad exigidos por el Ministerio de Educación Nacional. Asimismo, como se mencionó, la institución ha realizado una transformación a través del Proyecto Educativo de las Fuerzas Armadas (PefA), el Sistema Educativo de las Fuerzas Armadas (SEFA) y el Plan Estratégico del Sistema Educativo de las Fuerzas Armadas (PEse), en los cuales establece los lineamientos de educación de todas sus escuelas y programas académicos (Aristizábal et al., 2020).

Precisamente, en el PeI del Ejército Nacional se establece que una de las funciones misionales es la docencia, la cual se considera "como una actividad educativa específica, para facilitar que los miembros de la institución se apropien adecuadamente del conocimiento y ofrezcan soluciones a la comunidad" (Ejército Nacional de Colombia, 2016, p. 34). En este documento también se define sus propósitos:

- Generar en los estudiantes el pensamiento analítico, crítico y sistémico que facilita la construcción de nuevas estrategias y habilidades en el campo militar, la ciencia, la cultura y la profesión.

- Orientar los procesos de los estudiantes, velando por que cumplan con las características de la formación integral del militar.

- Vigilar que se innove en las prácticas pedagógicas y se garantice que el estudiante sea el actor principal del proceso educativo.

- Mantener actualizados los conocimientos, con el fin de brindar apoyo a los estudiantes en el campo militar.

- Mantener actualizados los cánones de acuerdo con los parámetros establecidos por el Ministerio de Educación Nacional (Ejército Nacional de Colombia, 2016, pp. 34-35).

De igual modo, se establece que el cuerpo docente está integrado por formadores, capacitadores e instructores, entre profesores militares y docentes civiles, y que esta labor exige conocimiento de los temas y las estrategias para enseñar. Ahora bien, con el fin de mejorar las habilidades pedagógicas y didácticas de los profesores militares se diseñan y realizan cursos para oficiales, suboficiales y soldados profesionales, en los cuales se les ofrece "preparación en el área de docencia, mediante el análisis y la reorientación de técnicas que busquen 
el mejoramiento en el desempeño de procesos de enseñanza y aprendizaje que permiten desenvolverse como docentes militares en los diferentes cursos" (Escuela de Armas y Servicios [EsACE], 2020). Específicamente, este curso se denomina Curso Preparadores de Instructores (CPI) y quien lo realiza "tiene la capacidad de ser instructor militar, contando con principios y valores que lo lleven a ser líder y orientador permanente de la doctrina militar" (ESACE, 2020).

También sobre la formación docente, Latorre (2020) sugiere la importancia de mejorar aspectos de la comunicación, la calidad en el diseño de investigación y el trabajo colaborativo en la formación de los docentes oficiales para fortalecer las competencias investigativas. Por su parte, Mantilla et al. (2020) consideran que la capacitación debe ser constante para que los docentes puedan guiar adecuadamente el proceso de aprendizaje de los estudiantes, mientras que para Palma et al. (2020) la formación militar tiene varios ejes que desarrollan el perfil profesional en la carrera militar.

Por lo anterior, se hace necesario indagar sobre el perfil pedagógico de los docentes e instructores de la Escuela de Aviación del Ejército Nacional y la Escuela Militar de Suboficiales “Sargento Inocencio Chincá”, ya que el centro del desempeño militar depende de los procesos de formación inicial y permanente durante la carrera, pero también porque actualmente las escuelas ofrecen diferentes programas y cursos a la sociedad civil, lo que hace necesario cumplir con los estándares de calidad exigidos por el sistema educativo colombiano.

\section{Métodos}

Los métodos cuantitativos se basan en la medición numérica y el análisis estadístico para establecer patrones de comportamiento y comprobar teorías (Hernández et al., 2014). En la investigación se decidió usar este método, ya que permitía, a través de una encuesta, extraer tendencias sobre los perfiles de los docentes-instructores en cuanto a títulos de pregrado y posgrado, años de experiencia docente, formación pedagógica a través de cursos cortos o de posgrados, necesidades de formación pedagógica y didáctica, producción académica, pertenencia a grupos de investigación, entre otros aspectos.

Participaron docentes e instructores de la Escuela Militar de Suboficiales "Sargento Inocencio Chincâ" y de la Escuela de Aviación del Ejército Nacional de Colombia, para un total de 89 docentes, lo cual representó el $10 \%$ de la 
población. La encuesta se realizó en línea y el análisis se desarrolló a través de estadística descriptiva. Con los resultados obtenidos se espera aportar información a las escuelas sobre su recurso humano docente que les permita plantear planes de formación, con el fin de cualificar las competencias pedagógicas y didácticas de sus instructores y docentes.

\section{Resultados}

A continuación, la tabla 1 muestra los resultados de la investigación de manera descriptiva. Como se mencionó, participaron 89 docentes-instructores, 57 de la Emsub y 32 de la Esave.

Tabla 1. Número de docentes/instructores caracterizados

\begin{tabular}{lcc}
\hline Escuela & $\begin{array}{c}\text { Número de docentes/ } \\
\text { instructores }\end{array}$ & Porcentaje \\
\hline $\begin{array}{l}\text { Escuela Militar de } \\
\text { Suboficiales "Sargento } \\
\text { Inocencio Chincá" }\end{array}$ & 57 & $64 \%$ \\
\hline $\begin{array}{l}\text { Escuela de Aviación del } \\
\text { Ejército Nacional }\end{array}$ & 32 & $36 \%$ \\
\hline
\end{tabular}

Fuente: elaboración propia.

De los docentes-instructores se estableció que el $80,9 \%$ son civiles y $19,1 \%$ son militares; de los militares, el $58,8 \%$ son oficiales y el $41,2 \%$ son suboficiales. El 80,9 \% de los docentes tienen contratación por hora cátedra y el $19,1 \%$ son de planta, lo cual significa que los de planta corresponden a los instructores militares.

En cuanto a la variable de género, se encuentra que el mayor porcentaje son hombres, con el 59,6\%, mientras que las mujeres representan el 40,4\%. Cuando se mira la edad, se encuentra que la mayoría de los docentes/instructores se encuentran en el rango de 26 a 36 años de edad (47,2\%), siguen los profesores en el rango de 37 a 46 (23,6\%), luego de 47 a 56 ańos de edad $(16,9 \%)$ y, por último, proporcionalmente, están los docentes-instructores que se encuentran entre los 18 y 25 años y los mayores de 56 años (tabla 2). 
Tabla 2. Nivel educativo de los docentes/ instructores

\begin{tabular}{lll}
\hline Nivel educativo & Número & Porcentaje \\
\hline Bachiller & 1 & $0,2 \%$ \\
Tecnólogo & 7 & $8 \%$ \\
Licenciado & 6 & $6,8 \%$ \\
Profesional & 75 & $85 \%$ \\
\hline
\end{tabular}

Fuente: elaboración propia.

Al preguntar por el nivel educativo se encontró que los docentes-instructores en su mayoría son profesionales (75) y en menor proporción tecnólogos (7), licenciados (6) y bachiller (1). Es de resaltar que los seis licenciados son de la Escuela Militar de Suboficiales "Sargento Inocencio Chinca’". En cuanto a los posgrados, la tabla 3 presenta las áreas del conocimiento de la especialización, maestría o doctorado.

Tabla 3. Área de conocimiento de los posgrados

\begin{tabular}{ll}
\hline Posgrado & Área \\
\hline Especializaciones & Ciencias de la astronomía \\
& Gerencia financiera \\
& Docencia universitaria \\
& Derecho \\
& Gerencia social \\
& Gestión de procesos psicosociales \\
\hline Maestrías & Gerencia de proyectos \\
& Dirección de empresas \\
& Dirección de negocios (MBA) \\
& Logística integral \\
& Ciencias astronomía \\
& Educación \\
& Educación ambiental \\
& Educación y cultura política \\
& Talento humano \\
& Ciencias penales \\
& Derechos Humanos \\
& Comunicación y educación con énfasis en cultura urbana \\
\hline Doctorados & Educación \\
& Dirección de proyectos \\
\hline
\end{tabular}

Fuente: elaboración propia. 
Igualmente, frente a la pregunta de si los docentes-instructores estaban realizando un posgrado actualmente, el $64 \%$ (57) dijo que no y el $36 \%$ (32) que sí. De estos últimos, 23 son de la Emsub y 9 de la Esave. Ahora bien, el hecho de que el $36 \%$ de los profesores de la muestra estén realizando un posgrado evidencia el interés por cualificar su quehacer docente y profesional, lo cual redunda en la calidad educativa de las escuelas.

Si bien son diversos los temas de los posgrados que están realizando, se resalta que son disciplinares, más que pedagógicos. Específicamente, se encuentran: 1 docente cursando una especialización en docencia universitaria, 8 profesores que está realizando maestrías relacionadas con el campo educativo y 1 profesor que hace un doctorado en educación, para un total de 10 docentes realizando posgrados en temas pedagógicos. La mayoría de estos docentes se encuentran en la EMsub.

Por otro lado, se preguntó sobre los posgrados finalizados, y se encontró que 10 tienen especialización, 6 tienen especialización y maestría, 8 maestrías y 3 tienen maestría y doctorado. De esos posgrados, 34 son en educación y pedagogía, el resto son en áreas disciplinares.

En cuanto al tipo de programas que enseńan en el Ejército, 50 aseguran que solo enseñan en carreras tecnológicas, 15 en cursos militares y 6 en carreras profesionales. Otros manifiestan que enseñan en varios tipos de programas: 1 en carreras técnicas-tecnológicas-especialización, 1 en cursos militares-carreras tecnológicas, 1 en cursos militares-especialización, 3 en cursos militares-carreras profesionales, 1 en cursos militares-tecnología, 2 en cursos militares-carreras técnicas-tecnológicas, 1 en cursos militares-tecnología-diplomados en convenio con universidades, 5 en cursos militares-tecnología-profesionales, y 2 docentes anotaron que enseñan inglés.

También se preguntó por su experiencia docente en el Ejército Nacional y se encontró que la mayoría se ubica en el rango de 1 a 5 años, con 59 docentes-instructores; le siguen 16 docentes que llevan de 6 a 10 años; luego, 8 de 11 a 15 años; 3 en el rango de 16 a 20 años; 1 en el rango de 21 a 26 años y 2 en el rango de 26 a 30 años, que serían los más antiguos (figura 1). 


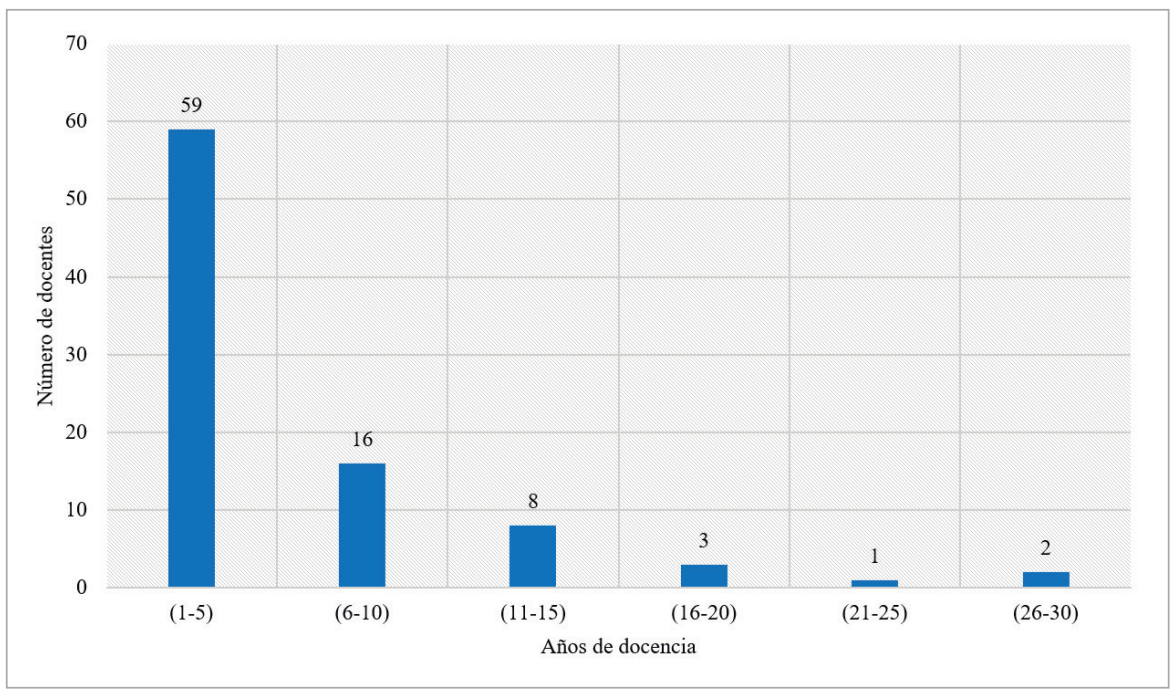

Figura 1. Años de experiencia docente en el Ejército Nacional.

Fuente: elaboración propia.

Posteriormente, se indagó acerca del tipo de programas e instituciones educativas en las que enseñan. Al respecto, los resultados muestran mucha diversidad, pues se encuentran docentes e instructores que enseñan en colegios, en programas técnicos laborales, en cursos de extensión, en carreras de pregrado (técnicos, tecnólogos y profesionales), en carreras de posgrado (especialización, maestría y doctorado) y 20 de ellos solo enseñan en el Ejército Nacional.

Las áreas de conocimiento en las que enseñan los docentes son muy diversas y la mayoría comparte diferentes áreas, que van desde ciencias básicas, administración, ambiente, ciencias sociales y humanas, hasta las ingenierías, la metrología, la seguridad y la defensa.

En cuanto a los años de experiencia docente, se encontró que la mayoría se ubica en el rango de 1 a 5 años (39), le siguen los de 6 a 10 años de experiencia (18) y, posteriormente, los de 11 a 15 años (13). En menor proporción se encuentran los que tienen experiencia mayor de 16 ańos (19).

Respecto a si los docentes e instructores han realizado cursos para perfeccionar sus competencias pedagógicas y didácticas, la mayoría dice que sí, con el 96,6 \% (86), muy pocos no han realizado cursos, el 3,4 \% (3). La mayoría 
de los cursos que han realizados son diplomados (13), cursos cortos (6), talleres (2), Cpr (1) y seminarios (1). Sin embargo, se destaca que los docentes han hecho más de un curso, pues muchos han realizado diplomados, cursos cortos, talleres, simposios, etc.

En cuanto a los modelos pedagógicos, el 80,9\% (80) dijo que sí los conocen, el 16,9 \% (8) afirmó que conoce algunos y el 2,2 \% (1) aseguró que no los conoce. La mayoría de los docentes e instructores señalan que conocen los modelos: constructivista, conductual y tradicional; en menor proporción los modelos: experiencial, constructivismo social, crítico y complejo. Se debe recordar que este último modelo es el que tiene el Ejército Nacional en sus proyectos educativos y en los documentos del Sistema Educativo.

Respecto a las didácticas, también se encuentra que los docentes-instructores combinan varias de ellas: clases magistrales con talleres prácticos, exposiciones, análisis de casos y mapas conceptuales. Algunos indicaron que solo trabajan por análisis de caso (1) o clase magistral (1) o clases prácticas (1).

Respecto al tipo de aprendizaje en el que se enfocan, la mayoría señaló el aprendizaje colaborativo y el aprendizaje basado en problemas. Posteriormente, indicaron el aprendizaje autónomo y el aprendizaje basado en proyectos. En el espacio del cuestionario destinado a otros, algunos señalaron aprendizaje por repetición, es decir, de memoria, y las tareas como parte de la apropiación del conocimiento.

En cuanto al uso de herramientas virtuales para las clases, el 75,3\% (78) dijo que las usa, frente al 2,2 \% (1) que manifiesta no usarlas; el 22,5 \% (10) las usa algunas veces. Frente a la pregunta sobre cuáles herramientas usan, los docentes-instructores contestaron bases de datos (generales, Proquest y Corte Constitucional), plataforma Blackboard y Classroom, Google Academic, videos, herramientas web, internet, celular, todas las herramientas ofimáticas, televisor y computador, Pixxon, Powtoon, Venngage, WebQuest, etc.

Respecto al tipo de evaluación que realizan, el $629 \%$ (56) manifestó que hace heteroevaluación, el 33,7 \% (30) coevaluación y el 3,4 \% (3) autoevaluación. Cuando se preguntó si cree que debe mejorar sus competencias pedagógicas y didácticas, el 77,5 \% (69) dijo que sí y el 22,5 \% (20) consideró que no. En cuanto a sus necesidades, manifestaron las siguientes: estrategias pedagógicas y didácticas, evaluación y tipos de evaluación, uso de las Tic y 
softwares, psicopedagogía, técnicas de aprendizaje, actualización disciplinaria y en temas militares, investigación científica, aprendizaje de las matemáticas, diversidad, innovación, manejo de bases de datos y técnicas para la oratoria.

También se indagó si pertenecen a un grupo de investigación: la mayoría expresó que no, con el 77,5 \% (69), en tanto que el restante 22,5 \% (20) dijo que sí pertenece. Respecto a si han realizado proyectos de investigación en los últimos cinco años, el 40,4 \% (36) de los docentes-instructores manifestaron que sí y el 59,6 \% (53) que no. Las áreas en que han realizado estos proyectos son diversas e incluyen: ingeniería, seguridad y defensa, salud, ambiente y biodiversidad, ciencias básicas, geociencia, aviación, Tic y ciencias sociales, humanas y educación, siendo esta última la de mayor énfasis.

\section{Discusión y conclusiones}

Las conclusiones de la investigación se centraron especialmente en los datos que más aportan a la caracterización del perfil pedagógico de los docentes e instructores de la EsAvE y la EMSUb. Los docentes e instructores en su mayoría son jóvenes, del género masculino, de hora cátedra y profesionales. De los 89 encuestados, solamente 6 son licenciados. Los programas de posgrado que han realizado se ubican más en áreas disciplinarias que en formación pedagógica y didáctica. Sin embargo, se observa que los docentes-instructores han estado preocupados por mejorar sus habilidades pedagógicas, pues en su mayoría han realizado diplomados, cursos cortos, talleres y simposios para tener mayor conocimiento y herramientas para enseñar. La experiencia como docentes en el Ejército Nacional y en general se encuentra principalmente en el rango de 1 a 5 años. Conocen sobre modelos pedagógicos, en especial el constructivismo, el conductismo y el tradicional. No conocen mucho sobre los modelos pedagógicos constructivismo social, experiencial, críticos y sistémicos-holísticos.

Las estrategias de enseñanza se centran en su mayoría en la clase magistral, análisis de casos y mapas conceptuales, en menor proporción las clases prácticas y talleres. El tipo de evaluación que más usan es la heteroevaluación y se enfocan en su práctica educativa en el aprendizaje colaborativo, el aprendizaje basado en problemas y el aprendizaje autónomo. La mayoría de los docentes-instructores usan las Tic en sus clases, desde bases de datos 
científicas, plataforma Blackboard, herramientas web y ofimáticas, televisor y computador hasta distintos softwares. Varios han tenido producción científica, desde artículos indexados hasta patentes. Por último, se observa el interés de los docentes e instructores de continuar su cualificación como docentes, al informar las necesidades de formación en temas como didácticas, uso de Tic, evaluación, psicopedagogía, investigación científica, manejo de bases de datos, actualización disciplinaria y militar, entre otros temas.

Las escuelas de formación militar, en especial la EsAvE y la EMSUB, sobre las cuales trata esta caracterización, ponen especial cuidado al logro de sus objetivos institucionales, uno de los cuales es la responsabilidad en la formación académica de los futuros militares. Por tanto, se hizo necesario realizar esta investigación para establecer, en primer lugar, el nivel profesional de los docentes-instructores encargados de enseñar los contenidos pertinentes en el aula. En segundo lugar, se dio a conocer la experiencia docente en el contexto militar, aspecto de mucha relevancia para la formación del alumno, ya que el docente militar debe tener claro ante todo la misión y la visión institucionales, aspectos característicos, especiales, únicos y diferentes a cualquier otra institución de formación en educación superior.

De igual manera, se verificó la actualización de los docentes-instructores, por cuanto se trata de un componente necesario en todo profesional que se dedique a enseñar. Así como el mundo sufre constantes cambios y transformaciones, el docente se debe hallar a la vanguardia para impartir los conocimientos de acuerdo con las actualizaciones de su campo disciplinar y las necesidades de formación de sus estudiantes. En este sentido, la verificación de esta actualización permitió dar a conocer si el docente-instructor se apropió de estos conocimientos para abordar herramientas y estrategias didácticas en el aula, dos elementos que deben ser permanentes para que se propicie una dinámica y lúdica en el aula, y por ende una apropiación asertiva de los contenidos. Además, si bien se estableció que los docentes-instructores tienen claridad sobre qué tipo o tipos de aprendizaje emplean, es necesario que se capaciten constantemente para que conozcan y se actualicen sobre los distintos tipos de aprendizaje, los cuales les aportarán mejores mecanismos y herramientas para poder abordar los contenidos curriculares de manera más efectiva frente a sus alumnos y de este modo cumplir a cabalidad con el logro de los objetivos. 
Hoy en día se hace necesario que toda institución y sus docentes trabajen de forma alterna: en modalidad presencial y virtual. Por lo tanto, no se puede considerar que las Tic son exclusivas de las instituciones virtuales ni una herramienta transitoria y/o esporádica. Es por ello que esta caracterización indagó sobre el uso de las Tic y permitió establecer que sí existe como un uso alterno, lo cual permite afianzar el conocimiento en el futuro militar, quien en su carrera profesional podrá desempeñarse de manera idónea.

\section{Referencias}

Aristizábal, J., Páez, C., \& Quintero, A. (2020). Procesos de calidad en la educación militar. Reflexiones en torno a la formación en investigación en la Escuela de Armas Combinadas. En L. Albornoz, A. Fernández, \& C. Betancur (Eds.), Práctica pedagógica en el Ejército Nacional de Colombia (pp. 159-180 297). Sello Editorial ESMIC. https://doi.org/https:// doi.org/10.21830/9789585241459.06

Comando de Educación y Doctrina [CEDOC]. (2020). Documentos rectores institución universitaria Colombia. https://www.cedoc.mil.co/cedoc.mil.co/normatividad/estatutos/ estatuto_general

Decreto 1070. (2015). Por el cual se expide el Decreto Único Reglamentario del Sector Administrativo de Defensa. Diario Oficial 49523.

Decreto 1495. (2002). Por el cual se reglamenta algunas disposiciones del Decreto Ley 1790 de 2000. Diario Oficial 44883.

Ejército Nacional de Colombia. (2016). Acuerdo No. 008 de 2016. Por medio del cual se aprueba y adopta el Proyecto Educativo Institucional de escuela militar de cadetes "General José Maria Córdova”. https://www.esmic.edu.co//recursos_user///Estatutos/PEI-ACUERDO008-FEB-19-2016.pdf

Escuela de Armas y Servicios [ESACE]. (2020). Curso Preparador de Instructores. http://esace. cemil.edu.co/index.php/programas/oferta-militar-internacional/curso-preparador-de-instructores/

González, H., \& Malagón, R. (2015). Elementos para pensar la formación pedagógica y didáctica de los profesores en la universidad. Reflection on Praxis, 17(2), 290-301. http://dx.doi. org/10.14483/udistrital.jour.calj.2015.2.a08

González, H., \& Ospina, H. (2013). El saber pedagógico de los docentes universitarios. Revista Virtual Universidad Católica del Norte, (39), 95-109. https://revistavirtual.ucn.edu.co/ index.php/RevistaUCN/article/view/429/878

Hernández, R. (2014). Metodología de la investigación. McGraw Hill.

Latorre, E. (2020). Diagnóstico de las necesidades de formación del personal de docentes oficiales del Ejército Nacional desde el modelo basado en evidencias. En L. Albornoz, A. Fernández, \& C. Betancur (Eds.), Práctica pedagógica en el Ejército Nacional de Colombia (pp. 181-210). Sello Editorial ESMIC. https://doi.org/https://doi.org/10.21830/9789585241459.07 
Mantilla, M., Loza, S., Casco, J., \& Saltos, N. (2020). Integración de las tecnologías de la información y comunicación para una educación militar innovadora. Revista Ibérica de Sistemas e Tecnologías de Informação, (29), 240-251. https://search.proquest.com/openview/d970a2d6687ef2d5e7b72e3979452be0/1?pq-origsite=gscholar\&cbl=1006393

Ministerio de Defensa Nacional. (2008). PESE Plan Estratégico del Sistema Educativo de las Fuerzas Armadas 2007-2019. https:/www.mindefensa.gov.co/irj/go/km/docs/Mindefensa/ Documentos/descargas/estrategia_planeacion/desa_capital/Pagina/PESE_FINAL.pdf

Ministerio de Defensa Nacional. (2010). SEFA Lineamientos curriculares. https://docplayer. es/136655835-Sefa-lineamientos-curriculares-fuerzas-militares.html

Moscoso, F., \& Hernández, A. (2015). La formación pedagógica del docente universitario: un reto del mundo contemporáneo. Revista Cubana de Educación Superior, 34(3), 140-144. http://scielo.sld.cu/scielo.php?script=sci_abstract\&pid=S0257-43142015000300011\&l$\mathrm{ng}=\mathrm{es} \& \mathrm{nrm}=\mathrm{iso}$

Palma, M., Garcés, F., Canalias, S., \& Albán, P. (2020). Análisis factorial de la escala de actitud hacia la estadística: estudio con estudiantes de las escuelas de formación de oficiales militares. Revista Ibérica de Sistemas e Tecnologías de Informaçâo, (29), 692-700. https://search. proquest.com/openview/0f5c06f94949d96003c85879c90cb52f/1?pq-origsite=gscholar\&cbl=1006393 